\title{
Regulation of Glucose, Fructose and Sucrose Catabolism in Rhodopseudomonas capsulata
}

\author{
By RALF CONRAD AND HANS G. SCHLEGEL \\ Institut für Mikrobiologie der Universität Göttingen und der Gesellschaft für Strahlen- und \\ Umweltforschung mbH, Grisebachstrasse 8, 3400 Göttingen, Federal Republic of Germany
}

(Received 2 November 1977)

\begin{abstract}
Regulation of glucose, fructose and sucrose catabolism was studied in Rhodopseudomonas capsulata grown under phototrophic conditions. The sequence of preference for the utilization of the sugar substrates was fructose, glucose, sucrose. The presence of a preferred substrate did not completely suppress the utilization of the less preferred. Glucose-6phosphate dehydrogenase, the key enzyme of glucose and sucrose catabolism, exhibited sigmoidal substrate saturation curves and was inhibited by phosphoenolpyruvate, whereas 1-phosphofructokinase, the key enzyme of fructose catabolism, exhibited hyperbolic substrate saturation curves and was not inhibited by phosphoenolpyruvate. Since phosphoenolpyruvate is a common intermediate of glucose, fructose and sucrose catabolism, the control of glucose-6-phosphate dehydrogenase may be responsible for the preferential utilization of fructose.
\end{abstract}

\section{INTRODUCTION}

Rhodopseudomonas capsulata is able to grow on glucose, fructose and sucrose under phototrophic as well as aerobic conditions. While glucose is degraded via the EntnerDoudoroff pathway (EDP), fructose is catabolized via fructose 1-phosphate and the EmbdenMeyerhof pathway (EMP) (Eidels \& Preiss, 1970; Conrad \& Schlegel, 1977a). Sucrose is hydrolysed in the cytoplasm and the resulting glucose and fructose are degraded via the EDP (Conrad \& Schlegel, 1978). Thus there are two separate sugar catabolic pathways in $R$. capsulata: one via fructose 1-phosphate and the EMP, and the other via fructose 6-phosphate, glucose 6-phosphate and the EDP. The enzymes of the EDP, except the sugartransport systems, are induced irrespective of which sugar the cells have been grown on (Conrad \& Schlegel, 1974, 1978). In contrast, the enzymes of the EMP, the phosphoenolpyruvate:fructose phosphotransferase system and 1-phosphofructokinase (EC 2.7.1.56), are only induced in the presence of fructose (Conrad \& Schlegel, 1974, 1977a).

The presence of two sugar catabolic pathways and their different inducibility raised the question of how the utilization of a pair of these sugars would be regulated. The present paper shows that fructose is utilized preferentially before glucose and sucrose. The role of glucose-6-phosphate dehydrogenase (EC 1.1.1.49) in the regulation of sugar catabolism is discussed.

\section{METHODS}

Rhodopseudomonas capsulata strain $\mathrm{Kb1}$, DSM 155, was obtained from the Deutsche Sammlung von Mikroorganismen, Göttingen, F.R.G. The composition of the growth medium has been described previously (Conrad \& Schlegel, 1977a). The bacteria were grown on glucose, fructose or sucrose (5 to $10 \mathrm{~mm}$ ) under phototrophic conditions and harvested during the exponential growth phase. The suspension of washed bacteria $(5 \mathrm{ml})$ was transferred to $200 \mathrm{ml}$ growth medium containing $20 \mathrm{~mm}$-phosphate and the sugar sub- 
strate as indicated, and incubated in a glass bottle $(250 \mathrm{ml})$ under an atmosphere of $\mathrm{N}_{2}$ at approximately 3500 lux and $30^{\circ} \mathrm{C}$. Samples were taken with a syringe. The concentrations of glucose and fructose in the growth medium were measured by a hexokinase-coupled spectrophotometric assay. For the determination of sucrose, the samples were incubated with $\beta$-fructosidase at $\mathrm{pH} 4.6$ and, after the hydrolysis of sucrose, the $\mathrm{pH}$ was adjusted to 7.6 and glucose and fructose concentrations were measured (detailed description by Boehringer). The preparation of bacterial extracts and the enzyme assays were done as described by Conrad \& Schlegel (1977a).

\section{RESULTS AND DISCUSSION}

\section{Growth on a mixture of glucose and fructose}

When $R$. capsulata that had been grown on fructose or glucose under phototrophic conditions was transferred to a mixture of glucose and fructose, growth was biphasic (Figs 1,2). Fructose was utilized preferentially but glucose utilization was not completely suppressed. After exhaustion of fructose, growth was retarded for about $30 \mathrm{~min}$ before continuing on the residual glucose. With fructose-grown cells, fructose was immediately utilized at the same differential rate as in a control culture on fructose alone (Fig. 1). The differential rate of glucose utilization remained low as long as fructose was present; after exhaustion of fructose, the differential rate increased immediately to the rate which was characteristic for growth on glucose alone. With glucose-grown cells, glucose was initially utilized at a high differential rate, while fructose was not utilized. However, as soon as the enzymes of fructose catabolism (phosphoenolpyruvate:fructose phosphotransferase system and 1-phosphofructokinase) were induced, the utilization of glucose was increasingly suppressed (Fig. 2). This observation indicated that it was a catabolite of fructose rather than fructose itself which suppressed glucose utilization.

\section{Regulation of glucose-6-phosphate dehydrogenase}

All the enzymes for the catabolism of glucose are also present in fructose-grown cells, except the glucose-transport system (Conrad \& Schlegel, 1974, 1977 $a, 1978$ ). Therefore, the suppression of glucose utilization by a catabolite of fructose can be explained by two mechanisms only: (i) repression of the glucose-transport system; (ii) inhibition of one or more enzymes of the glucose catabolic pathway. Our results did not point to a control mechanism via repression of the glucose-transport system: after exhaustion of fructose the utilization of glucose and growth continued rapidly, indicating that the glucose-transport system was fully induced at this time.

Glucose suppression could, however, be explained in terms of mechanism (ii) since glucose6-phosphate dehydrogenase, the key enzyme of the glucose degradative pathway, showed sigmoid substrate (glucose 6-phosphate) saturation curves (Fig. 3). Glucose-6-phosphate dehydrogenase is the enzyme of the EDP which is usually subject to regulation by effectors. In $R$. capsulata, the properties of this enzyme were similar to those of other allosteric glucose-6-phosphate dehydrogenases. The enzyme was inhibited by free ATP (Fig. 3) as in Pseudomonas aeruginosa (Lessie \& Neidhardt, 1967), Alcaligenes (Hydrogenomonas) eutrophus (Blackkolb \& Schlegel, 1968), P. fluorescens (Schindler \& Schlegel, 1969), $R$. sphaeroides (Ohmann, Borris \& Rindt, 1970) and Azotobacter beijerinckii (Senior \& Dawes, 1971); however, $1 \mathrm{mM}-\mathrm{ATP}$ was not inhibitory in the presence of $3.5 \mathrm{~mm}-\mathrm{MgCl}_{2}$. The enzyme of $R$. capsulata was sensitive to inhibition by phosphoenolpyruvate (Fig. 3) like the enzymes of Corynebacterium (Arthrobacter) autotrophicum strain 7c and Alcaligenes eutrophus (Tunail \& Schlegel, 1972), of Caulobacter crescentus (Shedlarsky, 1974) and of Azotobacter chroococcum, Azotobacter vinelandii and Zymomonas mobilis (Opitz, 1977). The Hill coefficient of the glucose-6-phosphate dehydrogenase of $R$. capsulata was $h=2 \cdot 13$, indicating positive co-operativity with respect to the substrate glucose 6-phosphate (Fig. 3). This co-operativity was increased in the presence of phosphoenolpyruvate ( $h=2.72$ to 2.91 ) but not in the presence of ATP $(h=2 \cdot 13)$. ADP, AMP, fructose 1-phosphate, fructose 6phosphate and fructose 1,6-bisphosphate were also tested (at $1 \mathrm{~mm}$ ) as possible inhibitors, 


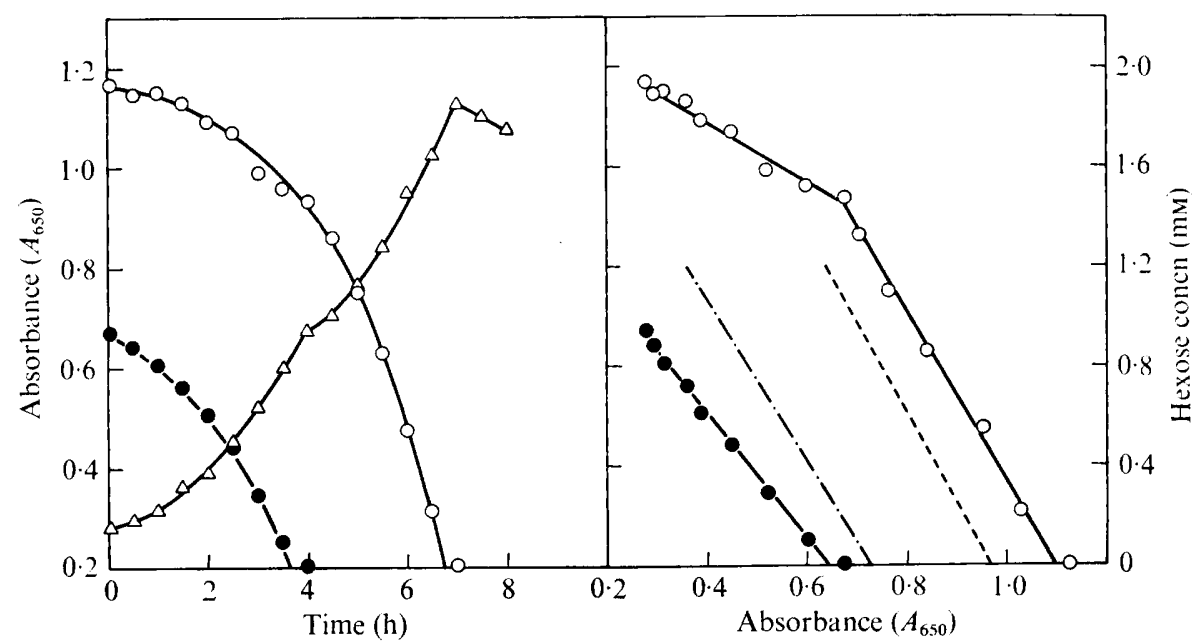

Fig. 1. Growth of fructose-grown $R$. capsulata on a mixture of $2 \mathrm{~mm}$-glucose and $1 \mathrm{~mm}$-fructose under phototrophic conditions: $\triangle$, absorbance; $O$, glucose concn; $O$, fructose concn; -.---, fructose concn in a control culture of fructose-grown cells on fructose; - - -, glucose concn in a control culture of glucose-grown cells on glucose.

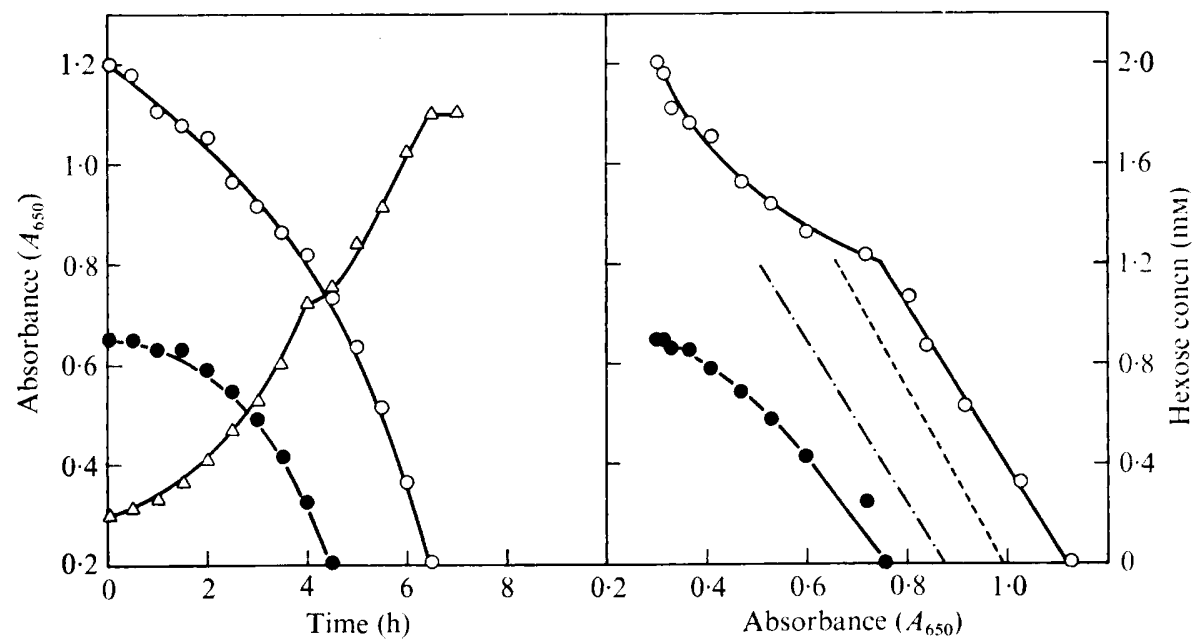

Fig. 2. Growth of glucose-grown $R$. capsulata on a mixture of 2 mM-glucose and 1 mM-fructose under phototrophic conditions: symbols as in Fig. 1.

but none of them inhibited the enzyme reaction significantly at a glucose 6-phosphate concentration of $1 \mathrm{mM}$.

The kinetic data suggested that glucose-6-phosphate dehydrogenase might be allosterically controlled by phosphoenolpyruvate in vivo. Phosphoenolpyruvate is a catabolite common to both the EDP and the EMP. One mole phosphoenolpyruvate is formed per mole hexose degraded in the EDP and two in the EMP. When glucose and fructose are catabolized simultaneously, the intracellular concentration of phosphoenolpyruvate might be expected to increase and to inhibit the glucose-6-phosphate dehydrogenase. This negative control would ultimately result in a decreasing glucose utilization and would thus explain why fructose was the preferred substrate of $R$. capsulata. 


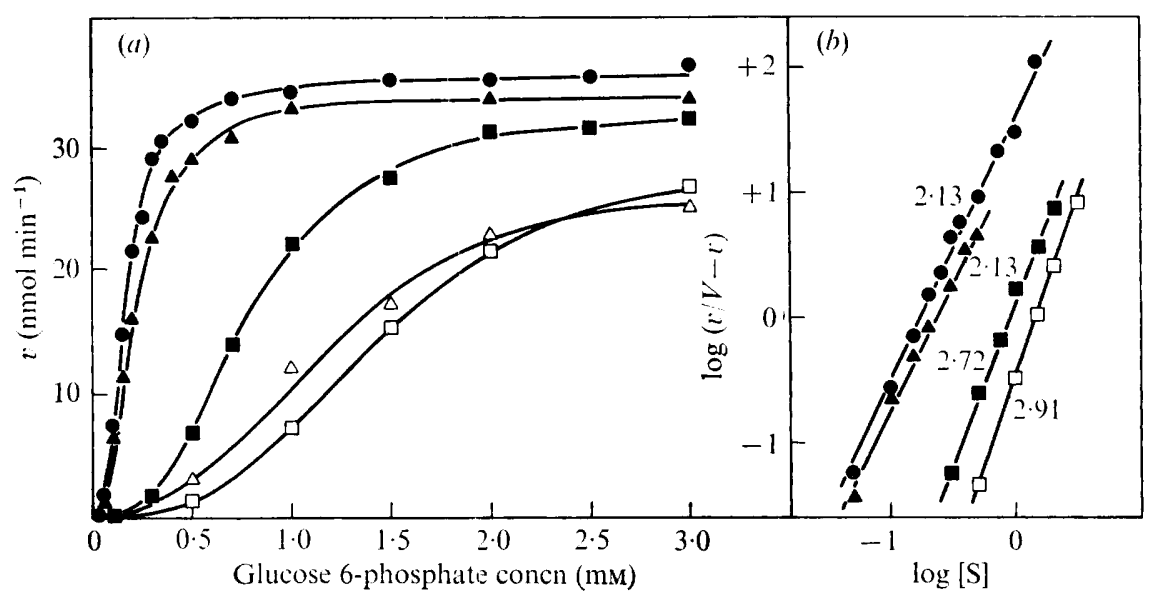

Fig. 3. Kinetic properties of the glucose-6-phosphate dehydrogenase. The assay mixture contained (in $1 \mathrm{ml}$ ): $45 \mu \mathrm{mol}$ triethanolamine/HCl buffer (pH 7.6), $0.45 \mu \mathrm{mol}$ NADP, Sephadex G-25treated extract (350 $\mu \mathrm{g}$ protein) and glucose 6-phosphate, ATP, phosphoenolpyruvate and $\mathrm{MgCl}_{2}$ as indicated. (a) Substrate saturation curves; $(b)$ Hill plots. Open symbols, without $\mathrm{MgCl}_{2}$; closed symbols, with $3.5 \mathrm{~mm}-\mathrm{MgCl}_{2} ; \mathbf{O}$, without effector; $\mathbf{\square}, \square$, with 1 mM-phosphoenolpyruvate; $\triangle$, with 1 mM-ATP.

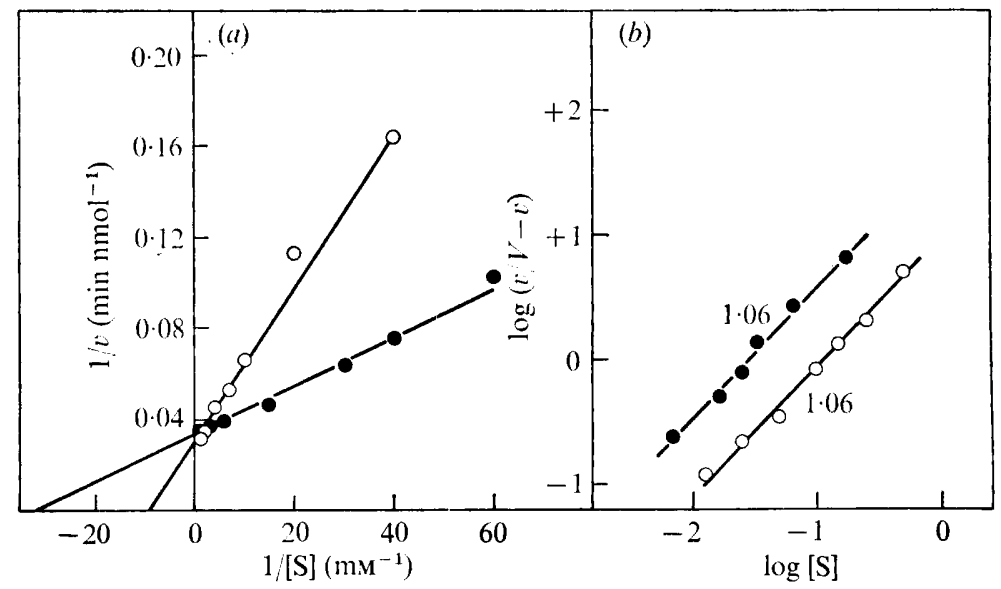

Fig. 4. Kinetic properties of the 1-phosphofructokinase. The assay mixture contained (in $1 \mathrm{ml}$ ): $39 \mu \mathrm{mol}$ triethanolamine/ $\mathrm{HCl}$ buffer $(\mathrm{pH} 8), 3 \mu \mathrm{mol} \mathrm{MgCl}_{2}, 6.65 \mu \mathrm{mol}$ reduced glutathione, $0 \cdot 25 \mu \mathrm{mol} \mathrm{NADH}, 0.18$ units aldolase, $2 \cdot 2$ units glycerolphosphate dehydrogenase, 26 units triose phosphate isomerase, Sephadex G-25-treated extract (140 $\mu \mathrm{g}$ protein), and either $0.75 \mu \mathrm{mol}$ ATP plus fructose 1-phosphate as indicated or $0.33 \mu \mathrm{mol}$ fructose 1-phosphate plus ATP as indicated. (a) Lineweaver-Burk plots; (b) Hill plots. O, $\mathrm{S}=\mathrm{ATP} ; \mathrm{O}, \mathrm{S}=$ fructose 1-phosphate.

\section{Regulation of 1-phosphofructokinase}

The assumption that the intracellular concentration of phosphoenolpyruvate controlled the degradation of glucose, but not fructose, was in accordance with the kinetic properties of the 1-phosphofructokinase. This is one of the key enzymes of fructose catabolism in $R$. capsulata. The 1-phosphofructokinase exhibited hyperbolic substrate (fructose 1-phosphate, ATP) saturation curves (Fig. 4) like all the 1-phosphofructokinases so far known from other bacteria: Enterobacter (Aerobacter) aerogenes (Sapico \& Anderson, 1969), Clostridium pasteurianum (du Toit, Potgieter \& de Villiers, 1972; von Hugo \& Gottschalk, 1974), 
Pseudomonas doudoroffii (Baumann \& Baumann, 1975) and P. putida (Bang, Baumann \& Sawyer, 1977). The 1-phosphofructokinase of $R$. capsulata showed apparent $K_{\mathrm{m}}$ values of $0.028 \mathrm{~mm}$ for fructose 1 -phosphate and $0.112 \mathrm{~mm}$ for ATP (Fig. 4). The Hill coefficient was $h=1.06$ for both substrates, indicating that they were not cooperatively bound to the enzyme. AMP, phosphoenolpyruvate, glucose 6-phosphate and fructose 6-phosphate did not act as inhibitors when tested at $1 \mathrm{~mm}$. ADP, one of the products of the enzyme reaction, was weakly inhibitory $(20 \%)$. The 1-phosphofructokinase was, therefore, unlikely to be a control point for fructose utilization. The second key enzyme of fructose degradation is the phosphoenolpyruvate:fructose phosphotransferase system. The number of components (membrane-associated and soluble) of the phosphotransferase system and their molecular interactions during the fructose translocation process have so far not been investigated in $\boldsymbol{R}$. capsulata. It is also an open question as to whether the phosphotransferase system constitutes a controlling site for the regulation of fructose breakdown. If the assumption is right, however, that phosphoenolpyruvate is only a substrate and not an inhibitor of the phosphotransferase system, fructose utilization would not be controlled by intracellular phosphoenolpyruvate, whereas glucose degradation via the EDP would be inhibited.

\section{Regulation of sucrose utilization}

Our conclusion that glucose-6-phosphate dehydrogenase is a control point in sugar catabolism was consistent with the results of a growth experiment with sucrose-grown cells. Like glucose, sucrose carbon is catabolized via the EDP in $R$. capsulata (Conrad \& Schlegel, 1978). We therefore examined whether the growth pattern of sucrose-grown cells on sucrose plus fructose was similar to that of glucose-grown cells on glucose plus fructose. In fact, sucrose utilization was suppressed in the same way as glucose utilization, as soon as fructose was catabolized simultaneously (Fig. 5). As sucrose-grown cells have a higher level of the phosphoenolpyruvate: fructose phosphotransferase system and 1-phosphofructokinase than glucose-grown cells (Conrad \& Schlegel, 1974, 1977a, 1978), the differential rate of fructose utilization increased faster and reached the rate of the control culture earlier than in glucosegrown cells.

When the experiment was repeated using a mixture of sucrose and glucose, sucrose utilization was suppressed to approximately one-third of the differential rate of a control culture on sucrose alone (Fig. 6). At the same time glucose was utilized at two-thirds of the differential rate of a control culture on glucose alone. Since the degradation pathway (EDP) is the same for sucrose and glucose, it was concluded that glucose carbon competed with sucrose carbon for the same enzymes.

\section{Additional remarks}

To our knowledge, the only two precedents for the preferential utilization of fructose before glucose are the glucose-utilizing mutant strain $\mathrm{H}^{16 \mathrm{G}^{+}}$of Alcaligenes eutrophus (Schlegel \& Trüper, 1966) and Thiobacillus strain A2 (Wood \& Kelly, 1977). In both these bacteria, growth on glucose is apparently limited by the glucose-transport system; this system may constitute the controlling site of the suppression of glucose utilization by fructose. Suppression of glucose utilization has also been observed in Arthrobacter crystallopoietes; in this bacterium, the glucose-transport system was repressed and inhibited by succinate (Krulwich \& Ensign, 1969).

Although our results point to a control of glucose utilization by allosteric regulation of glucose-6-phosphate dehydrogenase, inhibition of the transport systems of glucose and sucrose by a catabolite of fructose cannot be excluded. Mainly due to the complexity of membrane-bound enzyme systems, rather little is known about the control of sugar transport. However, the experimental evidence for an intricate regulation pattern of sugartransport activities is increasing (Kundig, 1974; Saier, Feucht \& Hofstadter, 1976; Postma \& Roseman, 1976; Kornberg \& Jones-Mortimer, 1977). In Escherichia coli, uptake has 


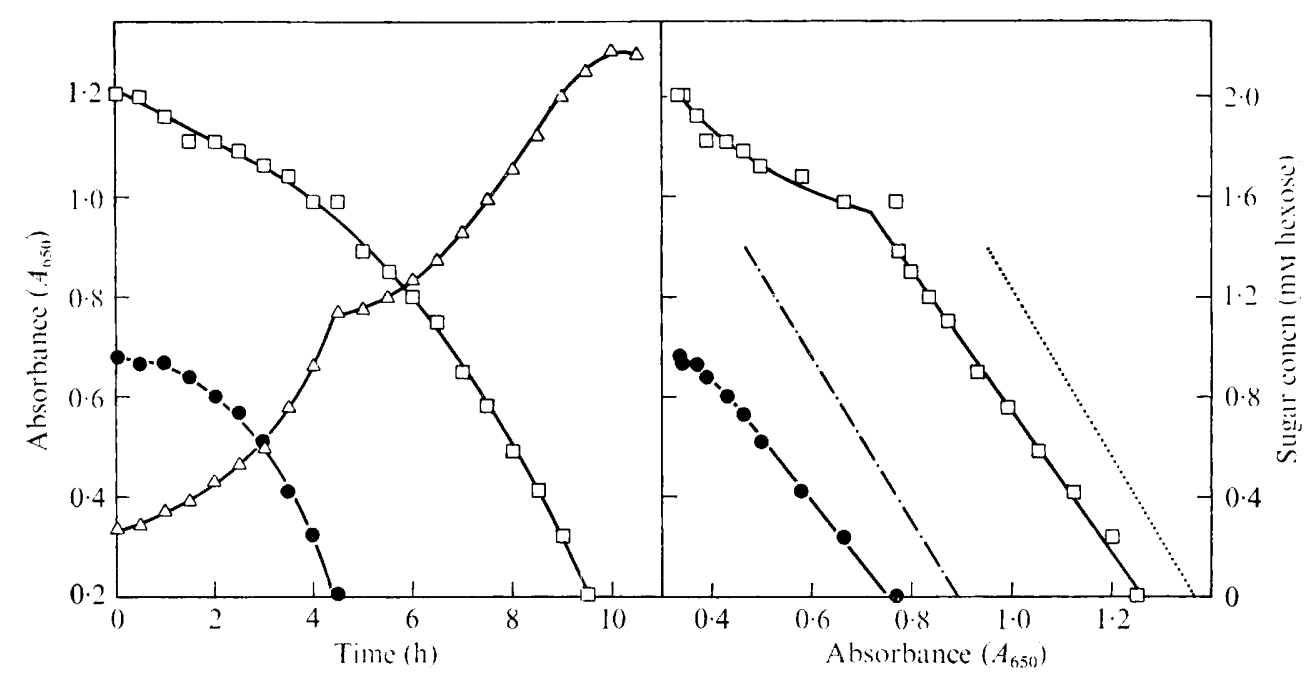

Fig. 5. Growth of sucrose-grown $R$. capsulata on a mixture of $1 \mathrm{~mm}$-sucrose and $1 \mathrm{~mm}$-fructose under phototrophic conditions: $\triangle$, absorbance; $\square$, sucrose concn; $\ominus$, fructose concn; $\cdots$, sucrose concn in a control culture of sucrose-grown cells on sucrose; -..--, fructose concn in a control culture of fructose-grown cells on fructose.

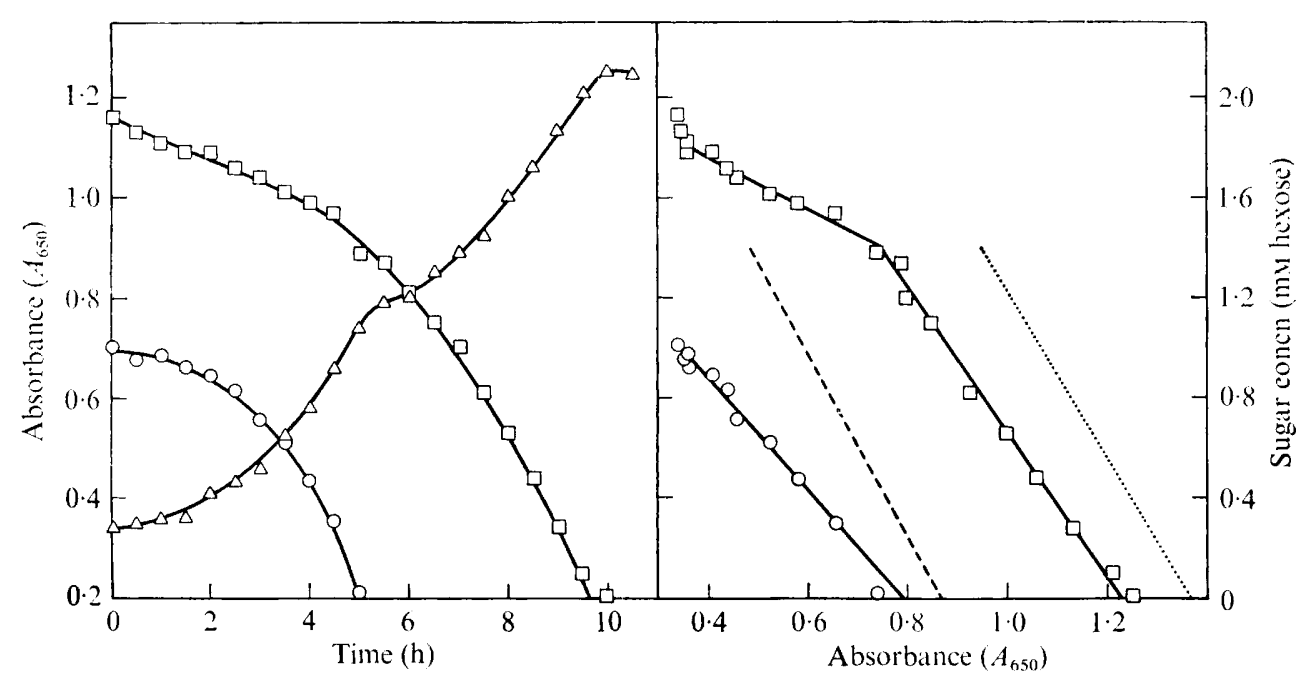

Fig. 6. Growth of sucrose-grown cells on a mixture of $1 \mathrm{~mm}$-sucrose and $1 \mathrm{~mm}$-glucose under phototrophic conditions: $\triangle$, absorbance; $\square$, sucrose concn; $O$, glucose concn; $\cdots$, sucrose concn in a control culture of sucrose-grown cells on sucrose; ---, glucose concn in a control culture of glucose-grown cells on glucose.

been shown to be the rate-limiting step during glucose catabolism (Herbert \& Kornberg, 1976), although this result was later questioned by Neijssel, Hueting \& Tempest (1977). In $E$. coli, however, glucose is the preferred substrate and not fructose; the phosphotransferase system-mediated uptake of fructose is inhibited by glucose or glucose 6-phosphate (Kornberg, 1972; Amaral \& Kornberg, 1975). Furthermore, the phosphoenolpyruvate:fructose phosphotransferase activity remains at a low level as long as glucose is present in the medium (Clark \& Holms, 1976). Inhibition of fructose transport by glucose has also been observed in Vibrio cholerae (Bag, 1974). However, there is so far no conclusive example for the 
reverse case, that glucose transport is inhibited by fructose or a fructose catabolite. In $R$. capsulata it is not yet known by what system glucose transport occurs, and the presence of a phosphoenolpyruvate phosphotransferase system for glucose also cannot be excluded. Future research should study both the glucose and fructose uptake systems in detail to elucidate whether the transport systems of $R$. capsulata constitute controlling sites in the regulation of sugar utilization in addition to the control of the glucose-6-phosphate dehydrogenase. A similar regulation pattern to that of $R$. capsulata would be expected for $R$. sphaeroides, since the pathways of glucose and fructose catabolism in these two organisms are identical, at least under phototrophic conditions (Conrad \& Schlegel, 1977b).

\section{REFERENCES}

Amaral, D. \& Kornberg, H. L. (1975). Regulation of fructose uptake by glucose in Escherichia coli. Journal of General Microbiology 90, 157-168.

BAG, J. (1974). Glucose inhibition of the transport and phosphoenolpyruvate-dependent phosphorylation of galactose and fructose in Vibrio cholerae. Journal of Bacteriology 118, 764-767.

Bang, S. S., Baumann, P. \& SaWyer, M. H. (1977). Properties of 1-phosphofructokinase from Pseudomonas putida. Canadian Journal of Microbiology 23, 721-725.

BaumanN, L. \& BaumanN, P. (1975). Catabolism of D-fructose and D-ribose by Pseudomonas doudoroffii. II. Properties of 1-phosphofructokinase and 6-phosphofructokinase. Archives of Microbiology 105, 241-248.

Blackkolb, F. \& Schlegel, H. G. (1968). Regulation der Glucose-6-Phosphat-Dehydrogenase aus Hydrogenomonas $\mathrm{H} 16$ durch ATP und $\mathrm{NADH}_{2}$. Archiv für Mikrobiologie 63, 177-196.

Clark, B. \& Holms, W. H. (1976). Control of sequential utilization of glucose and fructose by Escherichia coli. Journal of General Microbiology 95, 191-201.

ConRad, R. \& Schlegel, H. G. (1974). Different pathways for fructose and glucose utilization in Rhodopseudomonas capsulata and demonstration of 1-phosphofructokinase in phototrophic bacteria. Biochimica et biophysica acta 358, 221225.

ConRad, R. \& Schlegel, H. G. (1977a). Different degradation pathways for glucose and fructose in Rhodopseudomonas capsulata. Archives of Microbiology 112, 39-48.

CONRAD, R. \& Schlegel, H. G. (1977b). Influence of aerobic and phototrophic growth conditions on the distribution of glucose and fructose carbon into the Entner-Doudoroff and the EmbdenMeyerhof pathways in Rhodopseudomonas sphaeroides. Journal of General Microbiology 101, 277-290.

ConRad, R. \& Schlegel, H. G. (1978). An alternative pathway for the degradation of endogenous fructose during the catabolism of sucrose in Rhodopseudomonas capsulata. Journal of General Microbiology 105, 305-313.

EIdels, L. \& Preiss, J. (1970). Carbohydrate metabolism in Rhodopseudomonas capsulata: enzyme titers, glucose metabolism and polyglucose polymer synthesis. Archives of Biochemistry and Biophysics 140, 75-89.
Herbert, D. \& Kornberg, H. L. (1976). Glucose transport as rate-limiting step in the growth of Escherichia coli on glucose. Biochemical Journal 156, 477-480.

Hugo, H. von \& Gottschalk, G. (1974). Purification and properties of 1-phosphofructokinase from Clostridium pasteurianum. European Journal of Biochemistry 48, 455-463.

KorNBERG, H. L. (1972). Nature and regulation of hexose uptake of Escherichia coli. In The Molecular Basis of Biological Transport, Miami Winter Symposium, vol. 3, pp. 157-180. Edited by J. F. Woessner, Jr, and F. Huijing. New York, London: Academic Press.

KornBERG, H. L. \& Jones-Mortimer, M. C. (1977). The phosphotransferase system as a site of cellular control. Symposia of the Society for General Microbiology 27, 217-240.

KrulwiCh, T. A., \& Ensign, J. C. (1969). Alteration of glucose metabolism of Arthrobacter crystallopoietes by compounds which induce sphere to rod morphogenesis. Journal of Bacteriology 97, 526-534.

Kundig, W. (1974). Molecular interactions in the bacterial phosphoenolpyruvate-phosphotransferase system (PTS). Journal of Supramolecular Structure 2, 695-714.

Lessie, T. \& NeIDHARDT, F. C. (1967). ATP-linked control of Pseudomonas aeruginosa glucose-6phosphate dehydrogenase. Journal of Bacteriology 93, 1337-1345.

Neijssel, O. M., Hueting, S. \& Tempest, D. W. (1977). Glucose transport capacity is not the rate-limiting step in the growth of some wildtype strains of Escherichia coli and Klebsiella aerogenes in chemostat culture. FEMS Microbiology Letters 2, 1-3.

OhmanN, E., Borris, R. \& Rindt, K. P. (1970). Glucose-6-Phosphat-Dehydrogenase in autotrophen Mikroorganismen II. Die Regulation der Aktivität der Glucose-6-Phosphat-Dehydrogenase in Euglena gracilis und Rhodopseudomonas spheroides. Zeitschrift für allgemeine Mikrobiologie 10, 37-53.

OpITz, R. (1977). Die Verwertung organischer Substrate und die Regulation der Abbauwege bei Corynebacterium autotrophicum Stamm 19/-/x. Ph.D. thesis, Göttingen.

Postma, P. W. \& Roseman, S. (1976). The bacterial phosphoenolpyruvate:sugar phosphotransferase system. Biochimica et biophysica acta 457,213-257. 
Saier, M. H., JR, Feucht, B. U. \& Hofstadter, L. J. (1976). Regulation of carbohydrate uptake and adenylate cyclase activity mediated by the enzymes II of the phosphoenolpyruvate:sugar phosphotransferase system in Escherichia coli. Journal of Biological Chemistry 251, 883-892.

SAPICO, V. \& ANDERSON, R. L. (1969). D-Fructose 1-phosphate kinase and D-fructose 6-phosphate kinase from Aerobacter aerogenes. A comparative study of regulatory properties. Journal of Biological Chemistry 244, 6280-6288.

SCHINDler, J. \& Schlegel, H. G. (1969). Regulation der Glucose-6-Phosphat-Dehydrogenase aus verschiedenen Bakterien durch ATP. Archiv für Mikrobiologie 66, 69-78.

Schlegel, H. G. \& TRÜPER, H. G. (1966). Repression of enzyme formation in Hydrogenomonas strain $\mathrm{H}_{16} \mathrm{G}^{+}$by molecular hydrogen and by fructose. Antonie van Leeuwenhoek 32, 277-292.
Senior, P. J. \& Dawes, E. A. (1971). Poly- $\beta$ hydroxybutyrate biosynthesis and the regulation of glucose metabolism in Azotobacter beijerinckii. Biochemical Journal 125, 55-66.

SHEDLARSKY, J. G., JR (1974). Glucose 6-phosphate dehydrogenase from Caulobacter crescentus. Biochimica et biophysica acta 358, 33-43.

Toit, P. J. DU, Potgieter, D. J. J. \& Villiers, V. DE (1972). A study of the properties of 1-phosphofructokinase isolated from Clostridium pasteurianum. Enzymologia 43, 285-300.

Tunall, N. \& Schlegel, H. G. (1972). Phosphoenolpyruvate, a new inhibitor of glucose-6-phosphate dehydrogenase. Biochemical and Biophysical Research Communications 49, 15541560 .

Wood, A. P. \& Kelly, D. P. (1977). Heterotrophic growth of Thiobacillus A2 on sugars and organic acids. Archives of Microbiology 113, 257-264. 